\title{
A Two-Dimensional Theory of Health
}

\author{
Per-Anders Tengland \\ Health and Society Malmö University \\ 20605 Malmö, Sweden \\ per-anders.tengland@mah.se
}

\begin{abstract}
Slightly revised version of the article published as:
Tengland, P.-A. (2007). A Two-Dimensional Theory of Health.

Theoretical Medicine and Bioethics, 28(4); 257-284.
\end{abstract}

Please quote the original source

\begin{abstract}
The starting point for the contemporary debate about theories of health should be the holistic theory of Lennart Nordenfelt, claims George Khushf, not the refuted theory of Christopher Boorse. The present paper is an attempt to challenge Nordenfelt and to present an alternative theory to his and other theories, including Boorse's. The main problems with Nordenfelt's theory are that it is relativistic, that it leads to counter-intuitive results as to what goals can count as healthy, that it focuses on the wrong kind of abilities, that it makes measuring health extra difficult, and that it does not give us a sufficient account of health, at most a necessary one. The alternative theory proposed is two-dimensional. First, health is to have developed the abilities and dispositions that members of one's culture typically develop, and be able to use them, in acceptable circumstances; and second, health is to experience positive moods and sensations, the kinds that have internal causes. The theory solves the problems attached to Nordenfelt's theory by not being relative to the individual, by eliminating the goals in the definition, by giving an alternative interpretation of "ability," by making health easier to measure, and by adding the dimension of well-being that, together with health as ability, not only gives us a necessary, but also a sufficient, account of health.
\end{abstract}

KEY WORDS: Ability, basic ability, environment, goals, health, holistic theory, suffering, well-being

\section{Introduction}

I want to take up a challenge posed by George Khushf at the XIXth ESPMH conference. ${ }^{1} \mathrm{He}$ was surprised, he said, to see that the starting point for discussions on conceptual theories of health and disease is still, today, the theory of Christopher Boorse, a theory that has been thoroughly refuted over and over again. Instead, the new starting point for the contemporary discussion should be, he claimed, Lennart Nordenfelt's holistic theory of health. He then challenged Nordenfelt's theory. ${ }^{2}$ I shall not here summarize Khushf's critique of Nordenfelt, but try to develop a theory of health that goes beyond some of Nordenfelt's ideas. On the 
whole, however, I am sympathetic to Nordenfelt's theory, but, as I will show, there might be reasons to choose a theoretical variation on his holistic theme.

\section{Method}

I will ask what characteristics are necessary and jointly sufficient for a state of an individual to constitute health. I will not (primarily) ask what constitutes a certain level of health, be it optimal, good, or acceptable health, but what constitutes an increase (or a decrease) in health, what Bengt Brülde has called a "loose comparative approach." 3,4 However, sometimes we also need to make claims about "levels of health" (good health, acceptable health, etc.), so the theory proposed will take this into consideration as well. I will present an explication of the concept of health, starting from some basic intuitions about what health is, i.e., starting from our common linguistic understanding, but trying to sharpen these intuitions with the help of some other criteria. ${ }^{5}$

A number of criteria must be taken into account when discussing a definition of health: ${ }^{6}$ The first is the previously mentioned language criterion, which says that a definition should not differ too much from our ordinary use of the concept. The second is the value criterion (which can be seen as being part of the language criterion), which says that the definition should capture the positive value that the concept of health has. The third is the goal criterion, which says that the definition should make the goals of the healthcare sector (i.e., to promote health) reasonable. The fourth is the theory criterion, which says that it is preferable if the concept is presented as a conceptual theory or a principle which can help us pick out states or processes which fall under the concept, and which, moreover, help us explain why they belong there. The fifth is the homogeneity criterion, which states that it is preferable if health is defined in terms of a homogenous category. The sixth is the reliability criterion, which says that it is better if the definition is stated in observable terms, rather than in unobservable (for example, theoretical) ones. The seventh, the measurement criterion, is related to the previous criterion, and claims that it is preferable if the defining characteristics are measurable to some extent. The eighth criterion is the precision criterion, which says that the definition should be stated in precise and well-known terms. The last is the simplicity criterion, which says that the fewer exceptions or additions there are to the definition, the better.

It is impossible to accommodate all of these criteria at the same time, since some of them conflict. So, we have to compromise. The language criterion is, no doubt, the most important one, but given that language use differs widely, we need to make some stipulations using the other criteria. Thus, the result will be a suggestion as to how we should use the term given our practical and theoretical purposes in fields and professions like medicine, health care, rehabilitation, social work, psychotherapy, health promotion, and public health. A precise and well-argued definition should help us in trying to answer general questions about the purpose and goals of these professional areas. 


\section{Theories of health}

One can divide theories of health into (at least) four different monistic kinds, i.e., theories defined in terms of one kind of state or dimension: 1) health as functional normality, ${ }^{7,8} 2$ ) health as balance, ${ }^{9} 3$ ) health as ability, i.e., holistic theories, ${ }^{10-15}$ and 4) health as (subjective) well-being. ${ }^{16}$ There are also pluralistic theories of health, ${ }^{17-20}$ which means that they combine two or more states or dimensions that are said to constitute health.

My starting point will be the holistic theories (health as ability), since Nordenfelt is a representative of this tradition. I will also say a few words about health as balance, since it can be seen as a special case of the holistic theory, and I will discuss health as well-being, since I believe that well-being is an important part of the concept of health. Nothing will be said about the theory of health as functional normality, partly because of my focus in this paper, partly because I believe that the theory in its best formulations, the ones of Christopher Boorse and Jerome Wakefield, ${ }^{21-26}$ has been shown to be faulty as a general theory of health (and disease/disorder). ${ }^{27-36}$

In order to assess Nordenfelt's theory, I will compare it to some of the other holistic theories and show in what ways it is superior to them. But I will also try to show where it does not do so well, and why we need an alternative theory.

\section{Health as balance}

The theory of health as balance that will be presented here has a lot in common with the holistic theory of health. Ingmar Pörn has proposed that health is the balance between the individual's goals, the individual's repertoire (capacity, ability), and the environment in which the individual acts. ${ }^{37,38}$ In other terms, health is to have the repertoire necessary to reach one's chosen goals in the environment one is in. Health can therefore be compromised in three ways: the goals might be too ambitious (given the repertoire and the environment), the repertoire might be insufficient (given the goals and the environment), or the environment might become too harsh (given the repertoire and the goals). But this also means that an individual can gain increased health by three routes, i.e., by increasing her repertoire, changing her goals to ones that are more easily attainable, or "manipulating" the environment in order to make action easier. As a theory of adaptation it is fine, and it might, for example, be used as a conceptual basis for a theory of rehabilitation. ${ }^{39}$ As a theory of health it will not do. ${ }^{40}$

The theory does fulfill some of the above criteria, most importantly a) the theory criterion, since it is formulated as a theory that can help us determine if a state of an individual is a state of health, b) the value criterion, since the theory explains why it is good to have health, and c) the reliability and the measurement criteria, since this kind of balance is to some extent observable and measurable. 
It is still not suitable as a theory of health, primarily because it violates the language and the goal criteria. It is counter-intuitive (according to the language criterion), since the theory suggests that a person has decreased health only because the person has ambitious goals and cannot fulfill them. It is also counter-intuitive to claim that a person has decreased health just because a snow storm prevents her from reaching desired goals. It is similarly counterintuitive to claim that a person gains health by lowering her ambitions or by having her environment changed to make it easier to move around.

As for the goal criterion, it is an important goal of the healthcare system to increase the health of individuals, but generally not to change the environment (by installing elevators, lowering pavements, increasing street lightings, or make buses easier to access) or to help individuals lower their ambitions. ${ }^{41}$

\section{Health as ability}

Most holistic theories have the same starting point, as do theories of health as balance. Three components are central to these theories: ability, goals, and environment. What differentiates the various holistic theories are how these three components are envisaged. These theories focus on the holistic ability of the person. They are holistic in two senses. First, they start with the whole acting person, not with a reductionist focus upon parts or functions, like the theory of functional normality. Second, they are holistic because they take the environment into account, another factor that the theory of functional normality (more or less) leaves out of the picture. $^{42}$

The term "ability" will be used broadly to include abilities that are both intentional (to have the internal property needed to perform an action such as walking or pursuing a goal) and unintentional (acquiring a certain mental or physical state in certain circumstances, such as seeing in ordinary daylight).

There is an important distinction that has to be made in relation to a person's general ability. Nordenfelt has realized that there is a general problem with the notion of ability. ${ }^{43}$ There seem to be two kinds of general ability: health-related ones and non-health-related ones. Only the former should directly enter into a theory of health, as part of a definition of health. To be able to walk (assuming that it is a goal for the individual, or necessary for achieving one) is no doubt a health-related ability, i.e., to lack this ability is to lack some degree of health. However, not to be able to ride a bicycle does not necessarily indicate the absence of health, since the person might not have had the opportunity to learn to ride a bicycle. And, furthermore, even if the person learns to ride a bicycle we would not thereby say that she has gained health. There are many abilities of this kind, the absence of which does not constitute a reduction of health, and the existence of which does not (by definition) increase health, e.g., to swim, to read, or to drive a car. I will call abilities that require special training or education competence. ${ }^{44}$ Competences like the ones mentioned require health to be 
developed, but do not in themselves constitute health. ${ }^{45}$ So, how do we differentiate between these levels? Nordenfelt, for example, has tried to solve this problem. ${ }^{46} \mathrm{He}$ suggests that we differentiate between first order abilities (to reach goals) and second order ones. The first order abilities are the ones we at present have acquired. Having the second order ability is the ability to gain first order abilities. This is where health lies. A person might not be able to read, but if she is healthy she can learn to read, and she might not have an education, but if she is healthy (and is given the opportunity) she can acquire one. Thus, to be healthy is, according to Nordenfelt, to have the second order ability to reach vital goals.

\section{Environment}

We found that, according to the holistic theories, we cannot define health without taking the environment (in all its aspects) into consideration. The environment is in these theories seen as a background or platform for action. ${ }^{47}$ There are (at least) four ways to account for the environment in which the individual should be able to reach her goals: the environment the individual is in, ${ }^{48-50}$ the normal or standard environment, ${ }^{51}$ an acceptable environment, ${ }^{52}$ a variety of different environments (or "situations"), ${ }^{53}$ or different environments that can sometimes be demanding, but that are still acceptable. ${ }^{54}$

I believe that the last suggestion is the most plausible one. The first one has the same disadvantage as the theory of health as balance. If the environment the individual is in stops her from reaching her goals by putting obstacles in her way (if, for example, she is imprisoned), we will not in general say that there is something wrong with her health, a consequence that follows from this version of the theory, but rather that the environment is the cause of the inability. The same problem might appear if we choose the idea of a normal or standard environment. Mostly this assumption works well, but if the normal or standard environment is harsh and systematically hinders the individual from reaching her goals (for example, due to an ongoing civil war), we would not say that this failure is due to lack of health, as the theory suggests, but rather due to the (in this case normal) external circumstances. This is the reason why Nordenfelt introduced the idea of acceptable circumstances (environment). Thus, we only expect a healthy person to be able to act or to reach her goals if the circumstances are acceptable, i.e., such that most people are able to reach their goals in them. This, of course, involves a normative judgment, as we state what individuals should be able to do in the environment. But the idea of acceptable circumstances might also create problems. What if the environment is fully adjusted so as to compensate for every kind of state normally called "dysfunction" or "disability"? Then all people would be healthy, which is also counter-intuitive. Putting an elevator in a house does not make its inhabitants healthier just because it makes it easier for them to go out; at most, it compensates for their decreased health. 
So, instead we need a formulation of the environment component that says that health is to be able to reach one's goals or use one's abilities, in many circumstances, some rather demanding, but still acceptable. We expect people to be able to go to work in moderate snow fall but not in a snow storm, and we expect people to be able to go to work when the bus drivers are on strike, but only if there are other means of transportation available. We expect people who move to other countries to be able to reach some of their important goals, at least after having adapted to the new country, but only if the country in question gives reasonable opportunities for adaptation and goal fulfillment.

\section{Goals}

What, then, are the goals that a healthy person should be able to reach? There are at least four ways to formulate them. A healthy person should be able to 1) reach desired goals, ${ }^{55-57}$ 2) reach vital goals, ${ }^{58,59} 3$ ) reach the kind of goals that people typically reach in her society, ${ }^{60,61}$ or 4 ) do things people ordinarily do. ${ }^{62}$ Let us compare these ideas.

The first suggestion, to reach desired goals, has the same problem as we saw earlier in the theory of health as balance. According to the language criterion, it is counter-intuitive to conclude that a person has reduced health just because she has ambitious goals and cannot reach all of them. It is also problematic if the person's desired goals are extremely moderate, so moderate that a person could reach them no matter how "disabled." According to the theory, health would also change with the immediate change of desires, supposing, for example, that they went from easily attainable ones to ones that are hard to attain (or vice versa), a consequence that we might also question.

In order to solve these problems, Nordenfelt introduced the idea of "vital goals." These are the goals that contribute to the person's long-term happiness. Vital goals, as opposed to (only) desired ones, do not have to be consciously held, even though goals that turn out to be vital most likely are. Furthermore, vital goals are not always easily changed, since what makes us happy does not change easily. These goals have to do with our important values and preferences. However, our important goals can and do change over time, such as when someone might change her career, move to another part of the world, or pursue a new hobby.

The third and fourth suggestions above (to be able to reach typical goals, or to be able to do what people ordinarily do) will here be subsumed under one idea: to be able to reach goals typical in one's culture. ${ }^{63,64}$ This suggestion removes the individual relativism of the two first ideas, even if it might retain a cultural relativism (depending on the version of the theory used). The idea of reaching typical goals, thus, introduces the idea of normality into this theory. Note that the theory speaks of a very general kind of normality, e.g., goals such as holding a job, taking care of oneself, pursuing a hobby, and meeting with friends, which permits individuals to have very different goals at a specific level. Furthermore, as with several of these theories, it only requires the ability to reach typical goals, not actually 
reaching them. People might choose not to pursue goals that they could reach if they tried (or the environment could stop them from reaching the goals). Thus, disability (i.e., being unable to reach goals, in acceptable circumstances) is what determines if someone is unhealthy (and to what degree), and not unusual choices of goals.

We have now discussed the major elements in the holistic theories. Among these I find the theory of Lennart Nordenfelt to be the most convincing one. Let us therefore turn to a discussion and a critique of it.

\section{A critique of Nordenfelt's theory of health}

In the previous discussion of the terms ability, goals, and environment, we have seen how Nordenfelt uses these concepts. We have also seen that Nordenfelt, in some respects, has accommodated some of the problems of other similar theories. His own theory of health can be summarized in the following way: a person is fully healthy iff (if and only if) the person has the (second order) ability to reach all of her vital goals, assuming that the outer circumstances are acceptable. ${ }^{65,66}$ Any reduction in this general ability is a reduction in health. ${ }^{67}$ I will now try to show what some of the weaknesses of Nordenfelt's theory are. The theory fulfills many of our criteria to a high degree: value, since it shows why health is valuable by relating it to quality of life; homogeneity, since the theory is one-dimensional; reliability, since we can observe the abilities of individuals to some extent; precision, since all the concepts introduced are well-defined; and simplicity, since there are no exceptions or additions. It also fulfills some of the criteria to a lesser degree: language, goal, and measurement. It is in relation to these latter criteria that we find what is most problematic. The following points will be made: 1) the relativism of the theory leads to conflicts with the language and the goal criteria, 2) the idea of vital goals leads to counter-intuitive results as to what goals can count as healthy, 3) the theory's focus on second order abilities is counterintuitive, 4) the theory makes measuring health difficult, and 5) the theory does not give us a sufficient account of health, at most a necessary one.

First, two persons might have different degrees of health despite having the same mental and physical abilities, since their vital goals might be totally different, or they might have the "same" degree of health despite having different abilities, since their goals differ. This can be seen as being problematic according to the language criterion, since we often assess people's health in relation to their abilities, without necessarily taking their specific goals into account. Furthermore, a person with low ambitions, if we assume that these ambitions constitute her vital goals, will have good health, despite having a low degree of ability (compared with others). Likewise, a person with high ambitions, again assuming that these ambitions constitute her vital goals, might have low (or at least decreased) health despite having what we consider a high ability. This is not only problematic according to our language criterion, but also according to the goal and the value criteria. That a person has reduced health implies 
that her condition is bad in some sense and that she might be entitled to health care. However, this is not the case with the person with high ambitions (and high ability). The reverse goes for the person with low ambitions. She is in a bad state (because of her low ability) and is entitled to health care, despite having good health according to the theory.

I will, however, admit that our linguistic intuitions can go both ways in regard to some of these consequences. Consider a person that due to an accident ends up with a chronic illness or disability. This prevents her from living her life as before, i.e., from reaching her previous (vital) goals. Thus, her health has decreased. But, as she finds new goals in life, goals that are compatible with the "disability," we might agree with Nordenfelt that the person has gained health. Some people, however, would prefer to say that the person has compensated for her lack of health.

Second, consider another consequence of the theory. A pedophile who can reach his sexual goals - and let us assume that they in the long run lead to happiness (i.e., are vital) - without this hindering the attainment of other (vital) goals, must be deemed healthy. Only if this sexual urge comes into conflict with other vital goals (i.e., hinders the attainment of long-term happiness), goals such as staying out of jail, or pursuing a career, will his sexual preference be seen as constituting ill health. The same, of course, goes for other kinds of criminal or immoral behavior, like rape, murder, and torture. In other words: for Nordenfelt, a goal cannot in itself be considered a sign of ill health. Some people find these conclusions counterintuitive. We often call people with these kinds of desires mentally ill; states like these are considered bad for the person (no matter what the person herself thinks), and it is the goal for psychiatry (or psychotherapy) to try to change these kinds of desires.

Nordenfelt's answer is this: we have to differentiate between health and morality. The (active) pedophile is healthy but deeply immoral. The same goes for murderers, torturers, and rapists. Nordenfelt has a point, and I am not sure that our intuitions are wholly against Nordenfelt. Most theories uphold this distinction. ${ }^{68}$ Concluding that people are unhealthy only because they behave immorally is deeply problematic, since it rests solely on a value judgment. Every immoral act is then potentially an instance of ill health, a consequence that few would accept. So, if we do not agree with Nordenfelt, we need to make the distinction between health and morality in another way.

Third, as we have seen, Nordenfelt introduces the notion of second order ability to differentiate between health-related ability and non-health-related ability. To some extent this move succeeds, especially in relation to complex vital goals. It is not having a professional skill (assuming that this is a vital goal) that is part of health, but having the abilities necessary to acquire the professional skill. But the distinction also fails, mostly in relation to very basic vital goals. For example, in some cases it is not possible to make the distinction that Nordenfelt makes between first and second order abilities. To remember, to think, to walk, and to communicate can all be seen as very basic vital goals. Either we (at a certain age) have 
these abilities and are healthy, or we do not and are unhealthy to some degree. Here we have no use of the notion of "second order ability." Thus, health is in many cases having first order abilities to reach vital goals. A further remark, made by Brülde, is that if a person cannot acquire an ability that is vital to her, for example, read and write, then it is the fact that the person cannot read and write (despite having been given the adequate means to learn) that makes her unhealthy (in this respect), not primarily that she cannot learn to do so, even if this also has to be considered a reduction of health. ${ }^{69}$ This, again, indicates that health often (but not always) is situated at the first order level.

Fourth, the theory is also in conflict with the measurement criterion. The theory makes measuring health extra problematic. To measure health according to this theory we have to know two things: first, what the individual's vital goals are, and, second, if the person has the ability to reach these goals. This means that we cannot, for instance, just measure the person's ability to do things - not without first having asked the person what her important goals are. Furthermore, we might not even be sure that she knows what her vital goals are. The theory also makes repeated measurements of a person's health over time problematic since the person might not only have changed her ability over time, but also her vital goals. How does one compare the ability then to work as a salesperson with the ability now to be home and take care of one's children? So, this makes it impossible to measure any exact changes in health. Furthermore, the theory makes it impossible to compare the health of different individuals. $^{70}$

Finally, whether or not the previous remarks make us abandon Nordenfelt's version of the holistic theory, I contend that the holistic theories at most give us a necessary characteristic of health, not a sufficient one. What is lacking is well-being. ${ }^{71}$ Few theorists have focused on this dimension, with the exception of the WHO. According to their well-known 1948 definition, health is to be in a state of total physical, mental and social well-being. ${ }^{72}$ Even if few theoreticians explicitly define health in terms of well-being, there are quite a number of instruments for measuring generic health or treatment outcome that do include well-being as a health characteristic, something that supports my claim. ${ }^{73,74}$ I believe that our linguistic intuitions support this idea: ${ }^{75}$ to feel ill (in certain respects) is to have reduced health. Nordenfelt does not ignore this important aspect, but he assigns well-being a purely causal role, not a defining one. Well-being is a state that (by definition) contributes causally to our general ability, and suffering (ill-being), by definition, reduces our general ability. Thus, Nordenfelt claims that he takes this aspect of health into account. ${ }^{76}$ Two related arguments can be raised against this idea.

First, I believe that there is suffering that does not reduce the general ability, but that should still be seen as part of negative health. A person might be in a depressed mood or feel anguish, but might despite this be able to work, take care of herself, her home and her family, i.e., reach most of her vital goals. Nordenfelt's reply is this: it is likely that all suffering 
reduces the ability of the individual to some minimal extent - in fact, according to Nordenfelt's definition, it could not be considered suffering if it did not - and then the individual is at least minimally unhealthy. But I would still hold it likely that moderate ache, depression, or anxiety, for example, is compatible with reaching all one's vital goals (and, thus with full health). ${ }^{77}$ Despite being able to reach all vital goals, the person with moderate ache, depression, or anxiety is, I find it reasonable to say, in a state of reduced health.

Second, even if Nordenfelt is correct in his view that suffering always reduces ability to some minimal degree, it seems incorrect to claim that the health of the person is only minimally reduced (since the ability is only minimally reduced) when the suffering in fact is rather serious. ${ }^{78}$ It would be more accurate to claim that someone with severe suffering has low (mental) health, regardless of how much the ability of the person is reduced. Thus, there is often an asymmetry between the degree of suffering and the degree of disability it causes, and this asymmetry shows, I would argue, that we need to add the dimension of wellbeing/suffering to our definition of health.

\section{An alternative theory}

The alternative theory that will now be proposed consists of two dimensions, ability and wellbeing. ${ }^{79}$ I will present each dimension in turn.

\section{Health as ability}

I suggest an alternative formulation of the theory of health as ability. There are at least two options. One alternative is to accept, as suggested above, that health is the individual's ability to reach goals typical in one's society (or human goals). The other focuses on abilities and dispositions, and does not primarily take goals into consideration. In this latter view, health is having the abilities and dispositions that people typically develop. I have chosen to focus upon this latter variant. The reason is that it seems simpler and more direct to focus on the abilities, rather than on the goals. It also seems more in line with how we speak about health, for example, as having the (basic) ability to walk or to communicate, rather than reaching the (basic) goal to walk (to the store) or to communicate (with one's friends).

Thus, no specific generalized goal is assumed by this theory, but we should note that an ability is always an ability to something, i.e., to perform some action. ${ }^{80} \mathrm{I}$ will therefore assume here that the basic abilities, dispositions, etc., are abilities that are needed for all kinds of actions (or goal attainment). This means that every action requires a specific combination of abilities and dispositions. For example, in order to successfully perform most (if not all) actions we need to perceive reality (more or less) correctly and we need some motivational "drive." In other words, instead of being related to specific goals, like survival, reproduction, happiness, and cultural goods, the abilities are related to all goals people have. ${ }^{81}$

This theory, which comes in two versions, states that health is to have acquired the abilities, dispositions, and (some) "mental states" (abilities) that either a) human beings in 
general acquire, or that b) people typically acquire in their culture. Before settling the question of which version to choose, let us investigate what the different abilities are.

First, we have a number of (health-related) intentional abilities, i.e., abilities that we intentionally exercise, like grasping, lifting one's arm, walking, running, and jumping. A few mental examples are reasoning, trying to remember, imagining, and planning ahead, and some intentional social abilities are talking (to someone), playing (with someone), and living by the norms of one's society.

Some of our typical abilities are not exercised intentionally, but work "automatically." We can here call them unintentional; some examples are the ability to see (which is different from looking), and to hear (which is different from listening), to experience tactile sensations, to remember events (to some extent), and to be attentive (for some time). ${ }^{82}$ Note that most abilities make up dimensions, i.e., we can have more or less of them.

The healthy person also has the dispositions that people in general develop (and that often are "reactions" or automatic accommodations to changes in the environment). Some examples are to be able to experience emotions (e.g., love, anger, empathy, and sorrow), to be able to stay calm, to be able to control drives, and to have some resistance to stress.

Another important aspect of health is that of motivation, drive, energy, or vitality, i.e., that which makes us do things. This is sometimes referred to as the conative part of the mind, in contrast to the cognitive or the emotive (or affective) parts of the mind.

Finally, it seems that the healthy person also should have developed some other mental states or properties, the most important of which are to have a realistic and reasonably coherent apprehension of reality and to have developed some degree of self-confidence, i.e., a general sense (or belief) that one can handle different tasks and situations.

As already indicated, also in this theory we have to differentiate between health-related abilities and non-health-related abilities. The health-related ones I will call basic. These are, I suggest, the ones that individuals acquire (mostly in childhood and youth) just by growing up and living in a society. All other social, intellectual, or physical abilities, those that require special training (i.e., our competences), are non-health-related. ${ }^{83}$

In addition to having intentional and non-intentional abilities, motivation, and dispositions, and being in certain states, the healthy person has to have the ability to acquire competences of various kinds; in Nordenfelt's terms, she has to have the second order ability to acquire competences (i.e., first order abilities). The healthy person must be able to learn to read, write and count, ride a bicycle, and drive a car, i.e., to gain the competences that people normally gain when they have practiced for a while (sometimes a very long time).

An increase in any of the basic abilities, dispositions, etc., is an increase in health and a decrease in any of them is a decrease of health. We might, however, also want to discuss levels of healthh - acceptable or good health, for example. To have acceptable health is, then, 
to have a fair degree of the abilities and dispositions that are typical among one's fellow citizens, and to have excellent health is to have a high degree of these abilities.

Some further points have to be made. First, the more general an ability is, the more basic it is, and, I suggest, the more basic the ability is, the more important (in general) it is for health. To think, for example, is more basic than to imagine, to move the arm is more general than to throw a ball, and to experience emotions is more basic than to experience hope. Furthermore, some (basic) abilities are basic actions and basic actions are more 'basic' (in the health-related sense) than more complex actions. Performing a basic action means that when you are performing it you are not thereby performing some other action. ${ }^{84}$ For example, raising one's arm is a basic action, whereas waving is a non-basic action, since you wave by lifting and moving your arm (basic actions). So, in the health-related sense, raising one's arm is more basic than waving.

Second, we have to relate these abilities to sex and to age. What is typical of men and women differs to some extent, most importantly in the reproductive area. Therefore we have to differentiate between abilities and dispositions between men and women. As for age, since our abilities change as we grow up, from when we are infants, through childhood and adolescence, to when we are adults, we have to relate what is normal to a specific age. At a certain age, early adulthood, most of us have acquired (a fair degree of) more or less all of our basic abilities and dispositions (with some sex differences). Few basic abilities or dispositions are added after that, but if they typically are, they will be part of our health. Finally, when we grow old we lose some of our acquired abilities and dispositions. Here, I believe, we should accept that this is a reduction of health. Thus, we should not say that because reduced ability to keep one's balance is common among the old, this is still a healthy state. Every reduction of a basic ability is a reduction of health. Therefore, age relativity should only be applied to the ages where most of us acquire our health-related abilities.

Third, we need to take care of a potential problem: what if in the future most people (for genetic or environmental reasons) have lost the major part of some basic ability, e.g., their eye sight, would this still be an unhealthy state? According to the theory, it appears not to be unhealthy, since reduced eye-sight is then typical. The solution comes in two steps: first, we can claim that health is to have the abilities that human beings (in a society) typically have developed and attained through history or evolution. ${ }^{85}$ Thus, any reduction of such an ability or disposition developed over time, in the majority of the population, will constitute reduced health, but only - and here is the second step - if this negatively affects the attainment of quality-of-life goals typical in the society (or of humans in general). In other words, a (future) reduction of a typical ability is not considered a reduction of health if it does not affect the attainment of typical quality-of-life goals. For example, good hearing is a typically developed (non-intentional) ability of humans in all societies, so reduced hearing, however typical in the future, will be considered as a state of ill health, unless the reduction of hearing has no 
negative effect on the attainment of typical quality-of-life goals in one's society. Here, of course, we reintroduce one of Nordenfelt's important ideas, namely, his idea that health is (conceptually) related to quality of life (or happiness). Another point worth mentioning here is that any further future development of typical abilities in a society (or among humans in general) will still become part of people's health, even if they were not present in earlier generations. Imagine that in the future people will have developed some extra sensory perception, then lacking such an ability will be lacking an aspect of health, even if it does not now constitute decreased health.

Fourth, we have the question of utilizing our abilities. Not only do we have to have acquired these typical basic abilities, we also have to be able to use them, or be in these states, here and now. We can call this "executive capacity," or, as Brülde does, "performance capacity." 86 Thus, there are two ways in which we can be unhealthy; we can either lack an ability that we were never able to acquire, e.g., having no ability to walk because of a congenital defect, or we can have lost an ability that we had acquired temporarily (e.g., being incapable of getting out of bed because of a flu), or permanently (e.g., being blind because of an accident).

Finally, we have to add the environment clause that I argued for earlier. If the environment stops the individual from reaching her goals, then this does not count as ill health. We only expect healthy people to be able to use their acquired abilities and dispositions if the environment is acceptable, i.e., of a kind where most people can use their abilities and dispositions, even if we also expect healthy people to be able to cope with some environmental difficulties.

Let us now compare the version of the theory that defines health in terms of abilities typical of members of a society (or people), ${ }^{87}$ with the one that defines health in terms of abilities typical of human beings. The former theory is culture relative and the latter is not. It might be politically more correct to hold on to the first. In the end, the differences are minute, I believe. Basically, this is because the basic abilities in different societies are more or less the same. Abilities such as walking, talking, remembering, thinking, sleeping, keeping one's balance, and telling right from wrong, are common to all of us. There might, however, in some cases be differences. Imagine a society where it is as natural to swim as it is to walk. Not being able to swim would in such a society constitute some degree of ill health. This would not be the case in most other societies. As another example, using a computer is today a non-health-related ability. It is, rather, a competence. However, in the future it might be a health-related one, since we can imagine that all children in the future will interact with computers (which by then will be an integrated part of the environment) from a very early age. A person in that society will not be fully healthy if she cannot interact with computers. Notice also that having had the opportunity to learn does not come into play here, as with some other theories. Not being able to use a computer is having reduced health, as we now 
would say that not being able to walk is not having full health, no matter what the reason. The conclusion is, I believe, intuitively sound, since without the ability in question the person would be severely handicapped in this future society. As to choosing between the two versions of the theory, I will here without further argument accept the culturally relative one. $^{88}$

How does this theory solve the problem we found in Nordenfelt's theory? First, it is not relativistic on the individual level. All people's abilities are judged according to what is typical in that culture. This means, for example, that we can compare the health of people more easily, not first having to find out what their goals are. For the same reason, it will also be easier to measure health, since we can go straight to the abilities themselves, not having to ask about people's goals.

Second, goals do not enter into the definition in the same way as they do in Nordenfelt's, so we will not get the problem with someone having immoral goals that might turn out to be vital for the person, i.e., promote her long-term happiness. ${ }^{89}$ Note, however, that the theory still keeps health and morality conceptually apart, and that the question whether or not some kinds of ill health contribute to crime or other immoral actions is an empirical question. So, immoral persons might still be unhealthy, but not because of the crime itself, but rather because they lack some typical ability or disposition, which contributes to the illegal and immoral actions. The person might, for example, lack the ability to perceive reality correctly, lack empathy, or lack the ability to understand what is deemed morally right and wrong in their culture.

Third, when differentiating between health-related and non-health-related abilities we do not run into the problems that Nordenfelt does when he introduces the distinction between first and second order abilities. The distinction is instead made through differentiating basic abilities from competences.

It appears that this theory solves some of the problems with Nordenfelt's theory. But, what about the critique that Nordenfelt's theory (as well as others' theories) is at most necessary and not sufficient for having health? This problem can only be solved by including another dimension to the theory. No theory of health as ability does this, and, thus, they violate the language and goal criteria. We have to turn to a pluralistic theory despite this making the theory less homogenous. Health, I suggest, is having health-related abilities and having wellbeing, i.e., health has (at least) two dimensions - an idea we shall return to in the next section.

There are, however, still some problems that we must consider. How does this theory handle people who differ from most other people, like dissidents, eccentrics, and homosexuals, but who should be regarded as healthy according to our normal judgments? It is important to remember that health is not about normal behavior. Abnormal behavior, like committing crimes, breaking moral rules or rules of good conduct, or doing other unusual things, is not related to health unless it is the effect of some disability or lack of a typical 
disposition. But, then it is not the behavior as such, but the reduced ability or disposition that constitutes the reduction of health. Note that neither does supernormal behavior constitute increased or optimal health.

However, what if the lack of some ability takes the form of not being able to refrain from protesting against injustices, or not being able to form a heterosexual relationship? In the first case, there is no problem, since the dissident obviously has all of the abilities most people have. "Not being able," here, has to be interpreted in such a way that the person for strong moral reasons does not want to withhold her political views. However, if the inability to refrain from protesting is compulsive, one that the individual cannot control, then, of course, the person has some reduction of health (supposing that people typically can control such urges). ${ }^{90}$ As to the homosexual, it is obvious that she has the abilities of most people, and furthermore, even if she lacks the very specific disposition to form heterosexual relations, she has the more basic ability to form various other love relationships, including those with a partner, children, and parents, and, as we saw, having abilities (or dispositions) on this more basic level is more important for health than having less basic ones. ${ }^{91}$

Finally, let me address the question of "functional disabilities." It follows from the theory that people with "functional disabilities" are not fully healthy. A person who cannot walk is not fully healthy, despite the fact that she might be able to move around in a wheel-chair. This might be seen as problematic for the theory. Many "disabled" people today want to escape the stigma of being called disabled (or handicapped) or of being seen as having a disability. They can also feel perfectly healthy. Another example is people who are deaf and live in "deaf communities," where they can function perfectly well. ${ }^{92}$

I fully agree with the fact that persons with disabilities can feel healthy, and they can no doubt score high on the (health-related) well-being dimension. And, of course, they may score high on the quality-of-life dimension too. Moreover, they might have acquired many of the abilities and dispositions that people typically acquire (and be healthy in this respect). Still, I find it problematic to call people with disabilities fully healthy. This primarily has to do with motivating why we need to help people with disabilities. When we want to explain why we should give special support to those individuals, we need to resort to concepts like disability, dysfunction, handicap, or, in other instances, suffering or pain. Thus, in using such language we are already assuming that they are not fully healthy. I can see no other way to motivate the help needed than using these terms (and then the people in question are by definition not fully healthy).

\section{Health-related well-being}

Having developed one's basic abilities is a requirement for being healthy, but (subjective) well-being is also an important part of health. The term "well-being" is here used to refer to a subjective experience of some kind of feeling. The opposite of well-being I will here call 
suffering. However, not all well-being or suffering is health-related. So we have to differentiate between kinds of well-being. To make this distinction I will here use an idea similar to one put forward by Culver and Gert in their book Philosophy in Medicine. ${ }^{93}$ The idea is that well-being and suffering that are health-related have their immediate cause within the person herself. Well-being and suffering that are not health-related have outer sustaining causes, i.e., something outside the person that upholds the feeling. To clarify: this means that there is some external (immediate) causal influence that is necessary for the experience of well-being (in this sense). It is, of course, not sufficient.

As said, well-being always takes the form of a feeling. There are three kinds of feeling: sensations, i.e., feelings that are more or less localized somewhere in the body; moods, i.e., holistic non-localized feelings; and emotions, i.e., holistic non-localized feelings with a cognitive content consisting of beliefs and attitudes. ${ }^{94}$ The health-related well-being (and suffering) takes one of two forms: moods or sensations. ${ }^{95}$ All moods have immediate internal causes and are therefore health-related. Sensations can have both external and internal causes, and only those sensations that have immediate internal causes are health-related. Feeling vital, full of energy, concentrated, up to it, calm, strong, clean, and euphoric are some positive moods (and maybe sensations). Feeling tired, worn out, heavy, depressed, and anxious, are examples of health-related suffering, as are pain, ache, itching, throbbing, dizziness, nausea, or queasiness. $^{96}$ Taste and touch, on the other hand, are not health-related feelings, since they have external sustaining causes (that which we taste or touch). To repeat, health-related wellbeing is well-being in the form of moods or sensations that have an immediate internal cause, i.e., they are not upheld by an external sustaining cause.

The emotions, on the other hand, are feelings that do not count as health-related, since there is some external event or state of affairs that is necessary for the emotion to exist. For example, feeling happy because of some recent positive event, say the birth of one's child, is not having gained increased health, since the feeling is (partly) upheld by the event (through our beliefs about, and attitudes to, it). Neither is feeling unhappy because one has lost one's job a case of decreased health-related well-being. The loss of the job is the outer sustaining cause of this emotion.

The emotions might, however, involve some cognitive malfunctioning, e.g., include an irrational belief, but then the ill health will not count as suffering but as disability. There might also be a mismatch between cognitive content and "feeling," as when the feeling is not what one would expect given the beliefs and attitude of the person, for example, feeling happy when someone you love has died. But then it is not the feeling in itself that constitutes ill health, but the internal conflict between beliefs, attitudes, and feeling.

Finally, there is an important objection that has to be taken care of: does this not make (the mood) "existential anguish" (or anxiety) an example of ill health? Yes, it does, but I do not see this as a problem. I think that the basic idea that the experiencing of existential anguish is 
a state of health is faulty. Without having the space to develop these ideas in detail, the reason is this. Existential anguish, I believe, is a mental equivalent to physical pain. It has the purpose to warn us that there is some danger to the person's (authentic) existence. Existential anguish indicates that life (in some important sense), (mostly) unbeknownst to the person, is not lived well, i.e., that we are not living authentic lives, and that we need to reconsider what we do and how we live. ${ }^{97}$ Thus, existential anguish is a state of ill health. But it is not a disposition that we want to be without, as little as we want to be without the disposition to experience pain. ${ }^{98}$

\section{A two-dimensional theory of health}

We, thus, need a two-dimensional theory of health (fig. 1) in order to have a theory that better than Nordenfelt's theory meets some of the important criteria put up at the beginning, first and foremost the language, the value and the goal criteria.

\section{Figure 1}

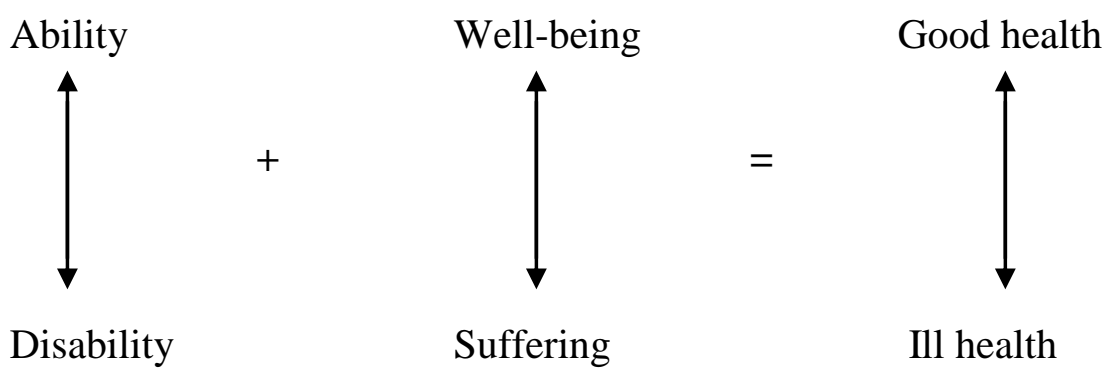

Figure 1: The two-dimensional theory illustrated, with the two dimensions ability and wellbeing that together make up the health dimension

The ability dimension, then, consists of all the individual's abilities, dispositions, etc., taken together, and the (subjective) well-being dimension consists of all the states of well-being taken together. ${ }^{99}$ An increase in any aspect of either of these dimensions is an increase in health, and a decrease in any aspect of these dimensions is a decrease in health. If we want to compare people's health, or if we want to try to establish degrees of health, the theory permits us to do so. Being acceptably healthy is having gained a fair degree of those abilities that people typically have acquired and to experience a fair degree of well-being in the healthrelated sense.

We should note that these two dimensions influence each other causally. Well-being, especially, tends to influence ability (as Nordenfelt claims). Thus, it is hard to believe that one can have severe pain but still have all one's abilities intact. Disability also influences wellbeing negatively, but most often quality-of-life-related well-being, rather than health-related 
well-being, for example, when one's disability makes one unhappy. We can, however, also imagine a person whose disability in the long run causes depression, i.e., health-related suffering.

\section{Conclusion}

How does this theory meet the adequacy criteria formulated in the beginning? On the positive side it fulfills the language criterion, the value criterion, and the goal criterion well. The reason for making the theory two-dimensional is to better fulfill these criteria. In ordinary language both ability and well-being are used for health, since both ability and well-being have a (direct or indirect) positive value for people, and disability and suffering have a negative value for them, and a primary goal of the healthcare system is to handle both disability and suffering. Measuring health, however, might be a problem. The theory is an improvement in relation to the theories where the individual's goals have to be taken into account, like Nordenfelt's theory, but adding the second dimension makes measuring health more complex. As to the reliability criteria, we can note that many abilities and dispositions are (more or less) observable, whereas feelings of (subjective) well-being or suffering are not. The theory, therefore, does less well according to this criterion. The major problem for the theory is homogeneity. Not only are there two dimensions, ability and well-being, but ability is not a homogenous category, since it consists of different kinds of abilities (intentional and non-intentional), of dispositions, of motivation, and of some acquired mental states (mainly correct perception and self-confidence). Others will have to judge how bad this is for the theory. 


\section{Acknowledgements}

I would like to thank Bengt Brülde and Lennart Nordenfelt for valuable comments on earlier versions of this paper. I would also like to thank the two anonymous reviewers for their challenging critique and useful suggestions.

\section{Notes}

${ }^{1}$ G. Khushf at the XIX ${ }^{\text {th }}$ European Conference on Philosophy of Medicine and Health Care in Barcelona, August 2005. Now published as “An agenda for future debate on concepts of health and disease," Medicine, Health Care, and Philosophy 10, no. 1 (2007): 19-27.

${ }^{2}$ See also G. Khushf, "What Is at Issue in the Debate about Concepts of Health and Disease? Framing the Problem of Demarcation for a Post-Positivist Era of Medicine," In Health, Science, and Ordinary Language, ed. L. Nordenfelt (Amsterdam: Rodopi press, 2001), pp. 123-169.

${ }^{3}$ B. Brülde, "On How to Define the Concept of Health: A Loose Comparative Approach," Medicine, Health Care and Philosophy 3 (2000): 305-308.

${ }^{4}$ B Brülde, "More on the looser comparative approach to defining 'health': A reply to Nordenfelt's reply," Medicine, Health Care and Philosophy 3 (2000):313-315.

${ }^{5}$ See L. Nordenfelt, On the Nature of Health: An Action-Theoretic Approach, 2nd revised edition, (Dordrecht: Kluwer, 1995), pp. 8-9 or Tengland, Mental Health: A Philosophical Analysis (Dordrecht: Kluwer Academic Publishers, 2001), pp 6-7.

${ }^{6}$ B. Brülde and P-A. Tengland, Hälsa och Sjukdom: En begreppslig utredning [Health and Illness: A Conceptual Investigation] Lund: Studentlitteratur, 2003.

${ }^{7}$ C. Boorse, "Health as a Theoretical Concept," Philosophy of Science 44 (1977):542-573.

${ }^{8}$ J.C. Wakefield, “The Concept of Mental Disorder. On the Boundary Between Biological Facts and Social Values," American Psychologist 4, no. 3 (1992): 373-388. Note that I assume that Wakefield's theory of disorder can be formulated as a positive theory of health. To my knowledge Wakefield has not put forward a theory of health.

${ }^{9}$ I. Pörn, “An Equilibrium Model of Health,” in Health, Disease and Causal Explanations in Medicine, eds. I. Lindahl and L. Nordenfelt (Dordrecht: Reidel, 1984), pp. 3-9.

${ }^{10}$ R. Dubos Mirage of Health: Utopias, Progress, and Biological Change, (New York: Harper and Row, 1959).

${ }^{11}$ C. A. Whitbeck, "Theory of Health," in Concepts of Health and Disease: Interdisciplinary Perspectives, eds.

S.L. Caplan, H.T. Engelhardt and J.J. McCartney (Reading, MA:Addison-Wesley, 1981), pp. 611-626.

${ }^{12}$ K. W. M. Fulford, Moral Theory and Medical Practice, (Cambridge: Cambridge U.P, 1989).

${ }^{13}$ G. Canguilhem, On the Normal and the Pathological, (New York: Zone Books, 1991).

${ }^{14}$ L. Nordenfelt, On the Nature of Health: An Action-Theoretic Approach.

${ }^{15}$ D. Seedhouse, Health (Chichester: John Wiley and Sons, 2001).

${ }^{16}$ WHO, Official Records of the World Health Organization, 2 (1948): 100.

${ }^{17}$ B. Brülde, Vad är hälsa? Några reflektioner kring hälsobegreppet [What is Health? Some Reflections about the Concept of Health] (Göteborg: Filosofiska institutionen, 1998). 
${ }^{18}$ B. Brülde, "On How to Define the Concept of Health: A Loose Comparative Approach."

${ }^{19}$ B. Brülde, "More on the looser comparative approach to defining 'health'."

${ }^{20}$ B. Brülde and P-A. Tengland, Hälsa och Sjukdom: En begreppslig utredning [Health and Illness: A

Conceptual Investigation] (Lund: Studentlitteratur, 2003).

${ }^{21}$ C. Boorse, “On the Distinction Between Disease and Illness," Philosophy and Public Affairs 5 (1975):49-68.

${ }^{22}$ C. Boorse, "What a Theory of Mental Health Should Be." Journal for the Theory of Social Behavior 6 (1976):61-84.

${ }^{23}$ C. Boorse, "Health as a Theoretical Concept."

${ }^{24}$ C. Boorse, "A Rebuttal on Health,” in What Is Disease? eds. J. Humber and R. Almeder. (Totowa, NJ: Humeana Press; 1997), pp. 3-134.

${ }^{25}$ C. Boorse, "Health as a Theoretical Concept."

${ }^{26}$ J.C. Wakefield, "Evolutionary Versus Prototype Analyses of the Concept of Disorder," Journal of Abnormal Psychology 108 (1999): 374-399.

${ }^{27}$ K. W. M. Fulford, Moral Theory and Medical Practice.

${ }^{28}$ J.C. Wakefield, "The Concept of Mental Disorder. On the Boundary Between Biological Facts and Social Values," Missing rest of citation

${ }^{29}$ S.I. Lilienfeld and L. Marino, "Mental Disorder as a Roschian Concept: A Critique of Wakefield's 'Harmful Dysfunction’ Analysis,” Journal of Abnormal Psychology 104, no.3 (1995): 411-420.

${ }^{30}$ D. Murphy and R.L. Woolfolk, “The Harmful Dysfunction Analysis of Mental Disorder,” Philosophy, Psychiatry and Psychology 7, no. 4 (2000): 241-252.

${ }^{31}$ D. Murphy and R. L. Woolfolk, "Conceptual Analysis versus Scientific Understanding: An Assessment of Wakefield's Folk Psychiatry,” Philosophy, Psychiatry and Psychology 7, no. 4 ( 2000): 271-293.

${ }^{32}$ L. Nordenfelt, On the Nature of Health: An Action-Theoretic Approach.

${ }^{33}$ L. Nordenfelt, “On Ability, Opportunity and Competence: An Inquiry into People's Possibility for Action,” in Contemporary Action Theory, Vol. I, eds. G. Holmström-Hintikka and E. Toumela (Dordrecht: Kluwer, 1997). ${ }^{34}$ L. Nordenfelt, Health, Science and Ordinary Language (Amsterdam: Rodopi Press, 2001).

${ }^{35}$ P-A. Tengland, Mental Health: A Philosophical Analysis (Dordrecht: Kluwer Academic Publishers, 2001).

${ }^{36}$ B. Brülde and P-A. Tengland, Hälsa och Sjukdom: En begreppslig utredning [Health and Illness: A Conceptual Investigation].

${ }^{37}$ I. Pörn, "An Equilibrium Model of Health."

${ }^{38}$ Another type of theory sees the balance as an internal one, for example as homeostasis, W. Cannon, Wisdom of the Body (New York: Norton, 1932), or as a balance between psychic forces, S. Freud, Orientering $i$ Psykoanalysen [Vorlesungen zur Einführung in die Psychoanalyse] (Stockholm: Natur och Kultur, 1976).

${ }^{39}$ See I. Pörn, “Health and Adaptedness,” Theoretical Medicine 14 (1993): 295-303.

${ }^{40}$ The same critique applies to David Seedhouse's theory Health (Chichester: John Wiley and Sons, 2001).

${ }^{41}$ We should, however, note that in some cases health care, for example in rehabilitation, involves a restructuring of the individual's goal profile (but not necessarily to a more modest one).

${ }^{42}$ See Boorse's "A Rebuttal on Health" for an account of his theory where he adds an environment component. 
${ }^{43}$ L. Nordenfelt, On the Nature of Health: An Action-Theoretic Approach.

${ }^{44}$ L. Nordenfelt, “On Ability, Opportunity and Competence: An Inquiry into People’s Possibility for Action,” in Contemporary Action Theory, Vol. I, eds. G. Holmström-Hintikka and E. Toumela (Dordrecht : Kluwer, 1997).

${ }^{45}$ A complication here is that there is a conceptual overlap between health and competence. An ability might be necessary both for health and for some competence. Compare being able to throw a ball (health) and to hit a target with a ball (competence).

${ }^{46}$ L. Nordenfelt, On the Nature of Health: An Action-Theoretic Approach.

${ }^{47}$ Note that this is different from discussing the environment as a causal factor for health or ill health.

${ }^{48}$ R. Dubos, Mirage of Health: Utopias, Progress, and Biological Change.

${ }^{49}$ I. Pörn, "An Equilibrium Model of Health."

${ }^{50}$ D. Seedhouse, Health.

${ }^{51}$ L. Nordenfelt, On the Nature of Health: An Action-Theoretic Approach.

${ }^{52}$ Ibid.

${ }^{53}$ C. A. Whitbeck, "Theory of Health," p. 611.

${ }^{54}$ B. Brülde and P-A. Tengland, Hälsa och Sjukdom: En begreppslig utredning [Health and Illness: A Conceptual Investigation].

${ }^{55}$ R. Dubos, Mirage of Health: Utopias, Progress, and Biological Change.

${ }^{56}$ I. Pörn, "An Equilibrium Model of Health."

${ }^{57}$ D. Seedhouse, Health.

${ }^{58}$ L. Nordenfelt, On the Nature of Health: An Action-Theoretic Approach.

${ }^{59}$ L. Nordenfelt, Health, Science and Ordinary Language.

${ }^{60}$ P.-A. Tengland, Mental Health: A Philosophical Analysis.

${ }^{61}$ P-A Tengland, "Social hälsa, vad för slags hälsa är det?” [“Social Health: What Kind of Health is that?”], in Hälsans sociala villkor [The Social Conditions for Health], eds. Ed. I. Kolfjord and L. Widding-Hedin (Lund: Bokbox Förlag, 2004).

${ }^{62}$ K. W. M. Fulford, Moral Theory and Medical Practice.

${ }^{63}$ Suggested in P.-A. Tengland, "Social hälsa, vad för slags hälsa är det?” ["Social Health: What Kind of Health is that?"].

${ }^{64}$ Fulford's idea about "ordinary doing" (see his Moral Theory and Medical Practice) can also be seen as a version of the theory of having the typical abilities of one's fellows in society, an idea we will return to.

${ }^{65}$ L. Nordenfelt, On the Nature of Health: An Action-Theoretic Approach.

${ }^{66}$ L. Nordenfelt, Health, Science and Ordinary Language.

${ }^{67}$ Notice that Nordenfelt also defines the notion of "acceptable health": a person has acceptable health iff (if and only if) the person has the (second order) ability to reach her basic vital goals assuming that the outer circumstances are acceptable (see On the Nature of Health: An Action-Theoretic Approach).

${ }^{68}$ See, for example, M. Pestana's Moral Virtue or Mental Health, Peter Lang Press, New York, 1998, where he explicitly argues for the importance of this distinction. 
${ }^{69}$ B. Brülde and P-A. Tengland, Hälsa och Sjukdom: En begreppslig utredning [Health and Illness: A Conceptual Investigation].

${ }^{70} \mathrm{We}$ should note that some of these problems are not unique to this theory.

${ }^{71}$ B. Brülde, Vad är hälsa? Några reflektioner kring hälsobegreppet [What is Health? Some Reflections about the Concept of Health] (Göteborg: Filosofiska institutionen, 1998).

${ }^{72}$ WHO, Official Records of the World Health Organization.

${ }^{73}$ I. McDowell and C. Newell, Measuring Health: A Guide to Rating Scales and Questionnaires 2nd. ed. (Oxford: Oxford U P, 1996).

${ }^{74}$ A. Bowling, Measuring Health: A review of quality of life measurement scales, 2nd ed. (Buckingham: Open University Press, 1999).

${ }^{75}$ That, of course, is the reason questions about well-being are included in the instruments measuring health.

${ }^{76}$ We should also note that Nordenfelt opens up for the possibility of a widening of the concept of health in this respect in his Health, Science and Ordinary Language. Notice also his discussion about "subjective health" in L. Nordenfelt, Quality of Life, Health and Happiness (Avebury: Aldershot, 1993).

${ }^{77}$ This, of course, is a question that has to be settled empirically.

${ }^{78}$ B Brülde, "More on the looser comparative approach to defining 'health'."

${ }^{79}$ It is a development of ideas found in Brülde 1998, 2000a-b, Brülde and Tengland 2003, and in Tengland 1998/2001, 2004, and 2006, “The Goals of Health Work: Quality of Life, Health and Welfare,” Medicine and Health Care and Philosophy 9, no. 2 (2006): 155-167.

${ }^{80}$ L. Nordenfelt, On the Nature of Health: An Action-Theoretic Approach.

${ }^{81}$ In general this works fine, but in the end we might still have to relate these goals to quality of life.

${ }^{82}$ We might equally well characterize some of these "abilities" as dispositions.

${ }^{83}$ For a similar idea about basic abilities see Brülde’s Vad är hälsa? Några reflektioner kring hälsobegreppet. [What is Health? Some Reflections about the Concept of Health.].

${ }^{84}$ L. Nordenfelt, On the Nature of Health: An Action-Theoretic Approach.

${ }^{85}$ See Wakefield, “The Concept of Mental Disorder: On the Boundary Between Biological Facts and Social Values" for a similar idea.

${ }^{86}$ B. Brülde, Vad är hälsa? Några reflektioner kring hälsobegreppet. [What is Health? Some Reflections about the Concept of Health.].

${ }^{87}$ Note that I am here not referring to sub-groups or sub-communities within a larger community, like the "gay community" or the "deaf community."

${ }^{88}$ One possible argument against this idea is that some culture or population might lack a basic ability common in other cultures or populations, say differentiating between certain colors, and we cannot then say that they lack health in this respect.

${ }^{89} \mathrm{We}$ have to accept, however, that a culture or people that have developed strong (basic) dispositions to display immoral behavior, say cruelty, are still fully healthy.

${ }^{90}$ Note that such a person would be much less morally praiseworthy, since this act of protest was not freely chosen (in full knowledge of its possible adverse consequences). 
${ }^{91}$ It might be suggested that nothing is as fundamental as forming heterosexual relations, since otherwise the species would not survive. That is true, but health in our sense is not defined biologically, or on the species level. It is not, then, necessary to say that having a heterosexual relation is more fundamental than having a romantic relationship or friendship. We should also remember that homosexuals have their reproductive abilities intact, and that some even have children.

${ }^{92}$ Remember that this is not the level of community that the theory speaks about.

${ }^{93}$ C. M. Culver and B. Gert, Philosophy in Medicine (Oxford: Oxford University Press, 1982).

${ }^{94}$ L. Nordenfelt, On the Nature of Health: An Action-Theoretic Approach.

${ }^{95}$ B. Brülde, Vad är hälsa? Några reflektioner kring hälsobegreppet. [What is Health? Some Reflections about the Concept of Health.].

${ }^{96}$ Note that it is not always easy to differentiate moods from sensations of a more holistic kind. However, for this purpose it is not very important, since both categories belong to health-related well-being.

${ }^{97}$ Anguish before having to choose can perhaps be seen as a special case of this.

${ }^{98}$ Nothing in this suggests that we need to treat the condition with drugs or the like. Psychotherapy, on the other hand, might be a good way to find out what is wrong in one's life.

${ }^{99}$ In reality we might have to include several dimensions, for example differentiating between abilities and dispositions that are physical and mental.

\section{References:}

Boorse, C. On the Distinction Between Disease and Illness. Philosophy and Public Affairs 5 (1975): 49-68.

Boorse, C. "What a Theory of Mental Health Should Be." Journal for the Theory of Social Behavior 6 (1976): 61-84.

Boorse, C. "Health as a Theoretical Concept." Philosophy of Science 44 (1977): 542-573.

Boorse, C. "A Rebuttal on Health." In What Is Disease? Edited by J. Humber and R. Almeder. 3-134 (Totowa, NJ: Humeana Press, 1997).

Bowling, A. Measuring Health: A review of quality of life measurement scales 2nd ed. Buckingham: Open University Press, 1999.

Brülde, B. Vad är hälsa? Några reflektioner kring hälsobegreppet. [What is Health? Some Reflections about the Concept of Health.] Göteborg: Filosofiska institutionen, 1998.

Brülde, B. "On How to Define the Concept of Health: A Loose Comparative Approach." Medicine, Health Care and Philosophy 3 (2000): 305-308.

Brülde, B. "More on the looser comparative approach to defining 'health': A reply to Nordenfelt's reply." Medicine, Health Care and Philosophy 3 (2000): 313-315.

Brülde, B. and P-A.Tengland. Hälsa och Sjukdom: En begreppslig utredning [Health and Illness: A Conceptual Investigation]. Lund: Studentlitteratur, 2003.

Canguilhem, G. On the Normal and the Pathological. New York: Zone Books, 1991.

Cannon, W. Wisdom of the Body. New York: Norton, 1932.

Culver, C. M. and B. Gert. Philosophy in Medicine. Oxford: Oxford University Press, 1982.

Dubos, R. Mirage of Health: Utopias, Progress, and Biological Change. New York: Harper and Row, 1959.

Freud, S. Orientering i Psykoanalysen [Vorlesungen zur Einführung in die Psychoanalyse]. Stockholm: Natur och Kultur, 1976.

Fulford, K. W. M. Moral Theory and Medical Practice. Cambridge: Cambridge U.P, 1989. 
Khushf, G. "What Is at Issue in the Debate about Concepts of Health and Disease? Framing the Problem of Demarcation for a Post-Positivist Era of Medicine." In Health, Science, and Ordinary Language. Edited by L. Nordenfelt. 123-169 (Amsterdam: Rodopi press, 2001).

Khushf, G. "An agenda for future debate on concepts of health and disease." Medicine, Health Care and Philosophy 10, no. 1 (2007): 19-27.

Lilienfeld, S.I. and L. Marino. "Mental Disorder as a Roschian Concept: A Critique of Wakefield's 'Harmful Dysfunction' Analysis.” Journal of Abnormal Psychology 104, no.3 (1995): 411-420.

McDowell, I and C. Newell. Measuring Health: A Guide to Rating Scales and Questionnaires, $2^{\text {nd }}$ ed. Oxford: Oxford U P, 1996.

Murphy, D. and R.L. Woolfolk. "The Harmful Dysfunction Analysis of Mental Disorder." Philosophy, Psychiatry and Psychology 7, no. 4 (2000): 241-252.

Murphy, D. and R. L. Woolfolk. "Conceptual Analysis versus Scientific Understanding: An Assessment of Wakefield's Folk Psychiatry." Philosophy, Psychiatry and Psychology 7 , no. 4 (2000): 271-293.

Nordenfelt, L. Quality of Life, Health and Happiness. Avebury, Aldershot, 1993.

Nordenfelt, L. On the Nature of Health: An Action-Theoretic Approach, Second revised edition. Dordrecht: Kluwer, 1995.

Nordenfelt, L. "On Ability, Opportunity and Competence: An Inquiry into People's Possibility for Action.” In Contemporary Action Theory, Vol. I, Edited by G. HolmströmHintikka and E. Toumela. Kluwer, Dordrecht, 1997.

Nordenfelt, L. Health, Science and Ordinary Language. Amsterdam: Rodopi Press, 2001.

Nordenfelt, L. "On the Evolutionary Concept of Health: Health as Natural Function." In Dimensions of Health and Health Promotion. Edited by L. Nordenfelt and P-E. Liss. Amsterdam: Rodopi Press, 2003.

Pestana, M. Moral Virtue or Mental Health. Peter Lang Press, New York, 1998.

Pörn, I. "An Equilibrium Model of Health." In Health, Disease and Causal Explanations in Medicine. Edited by I. Lindahl and L. Nordenfelt. 3-9 (Dordrecht: Reidel, 1984).

Pörn, I. "Health and Adaptedness." Theoretical Medicine 14 (1993): 295-303.

Seedhouse, D. Health. Chichester: John Wiley and Sons, 2001.

Tengland, P-A. Mental Health: A Philosophical Analysis. Dordrecht: Kluwer Academic Publishers, 2001.

Tengland, P-A. "Social hälsa, vad för slags hälsa är det?" ["Social Health: What Kind of Health is that?"]. In Hälsans sociala villkor [The Social Conditions for Health]. Edited by I. Kolfjord and L. Widding-Hedin.Lund: Bokbox Förlag, 2004.

Tengland, P-A. "The Goals of Health Work: Quality of Life, Health and Welfare." Philosophy, Medicine and Health Care: A European Journal 9, no. 2 (2006): 155-167.

Wakefield, J.C. "The Concept of Mental Disorder. On the Boundary Between Biological Facts and Social Values." American Psychologist 4, no. 3 (1992): 373-388.

Wakefield, J.C. "Evolutionary Versus Prototype Analyses of the Concept of Disorder." Journal of Abnormal Psychology 108 (1999): 374-399.

Whitbeck, C. "A Theory of Health." In Concepts of Health and Disease: Interdisciplinary Perspectives. Edited by S.L. Caplan, H.T. Engelhardt and J.J. McCartney. 611-626 (Addison-Wesley, Reading, Massachusetts, 1981).

WHO. Official Records of the World Health Organization, 2 (1948): 100. 